\title{
Marchés et quasi-marchés dans le financement de l'éducation
}

\section{Le cas de l'Angleterre}

Market and quasi market in education funding. England - case study

Mercados y casi-mercados en la financiación de la educación. El caso de

Inglaterra

\section{Anne West}

Traducteur : Jérôme Quintana

\section{(2) OpenEdition}

1 Journals

\section{Édition électronique}

URL : http://journals.openedition.org/ries/3683

DOI : $10.4000 /$ ries.3683

ISSN : 2261-4265

Éditeur

Centre international d'études pédagogiques

\section{Édition imprimée}

Date de publication : 15 avril 2014

Pagination : $57-67$

ISBN : 978-2-85420-603-6

ISSN : 1254-4590

\section{Référence électronique}

Anne West, «Marchés et quasi-marchés dans le financement de l'éducation 》, Revue internationale d'éducation de Sèvres [En ligne], 65 | avril 2014, mis en ligne le 15 avril 2016, consulté le 06 janvier 2020. URL : http://journals.openedition.org/ries/3683 ; DOI : 10.4000/ries.3683

Ce document a été généré automatiquement le 6 janvier 2020 .

(c) Tous droits réservés 


\title{
Marchés et quasi-marchés dans le financement de l'éducation
}

\author{
Le cas de l'Angleterre \\ Market and quasi market in education funding. England - case study \\ Mercados y casi-mercados en la financiación de la educación. El caso de \\ Inglaterra
}

\section{Anne West}

Traduction : Jérôme Quintana

1 La mise en place de politiques néolibérales dans divers pays du monde occidental a redéfini le rôle de l'état en matière d'offre de services sociaux. Le néolibéralisme " cherche à intégrer tout acte humain dans un marché » et, de manière significative, là où les marchés n'existent pas, il faut les créer (Harvey, 2005).

2 L'influence des idées néolibérales est manifeste dans les politiques éducatives mises en place en Angleterre par les gouvernements conservateurs de 1979 à 1997, et poursuivies, en y apportant quelques modifications, par les travaillistes de 1997 à 2010, puis étendues par la coalition gouvernementale entre conservateurs et libérauxdémocrates, à partir de 2010.

3 L'éducation a été au premier plan des changements apportés à l'offre de services sociaux en Angleterre. De fait, ces changements, intervenus au cours des dernières décennies, s'apparentent à un changement de paradigme. Dans l'éducation en milieu scolaire, qui comprend les écoles et les classes maternelles (principalement pour les enfants âgés de 3 à 4 ans), les écoles primaires (pour les enfants de 5 à 11 ans) et les établissements secondaires (pour les élèves de 11 à 16 ou 18 ans), il existe un « quasimarché » de l'éducation, où les parents peuvent choisir les établissements selon leur préférence et où l'argent suit les élèves dans l'établissement qu'ils fréquentent (c'est une forme de mécanisme de financement axé sur la demande). Il existe un type similaire de quasi-marché dans les établissements de l'enseignement post-obligatoire qui forment les jeunes de 16 à 19 ans. 
4 Dans l'enseignement supérieur, des changements radicaux ont eu lieu. Les fonds publics affectés à l'enseignement étaient par le passé versés directement aux universités. Mais aujourd'hui, les étudiants paient des frais de scolarité dont le montant a été multiplié par neuf depuis la fin des années 1990. Les étudiants ont la possibilité de contracter auprès d'un organisme public des emprunts qui couvrent les frais de scolarité et les frais d'entretien et de coût de la vie (maintenance loan). Ces emprunts sont remboursés une fois que l'étudiant a obtenu son diplôme universitaire et que son revenu annuel atteint un niveau déterminé.

5 Les idées néolibérales ont ainsi influencé la politique éducative, mais de manière différente selon les niveaux d'enseignement. Néanmoins, dans tous les cas, on a introduit ou amplifié une logique de marché. Dans cet article, nous nous intéressons plus particulièrement au financement de l'éducation au niveau préscolaire, primaire, secondaire (collège et lycée) et dans le supérieur.

\section{L'enseignement préscolaire}

6 L'enseignement préscolaire en Angleterre est dispensé dans différents cadres. Le système est complexe, tant sur le plan des établissements qui dispensent l'offre que sur celui du financement. Pour les enfants âgés de moins de 3 ans, l'enseignement et les structures d'accueil destinés à la petite enfance (early childhood education and care) sont normalement gérés par le secteur privé, qu'il soit à but lucratif ou non. Les parents paient pour que leurs enfants aient accès à ces structures, et il est important de souligner que le montant des frais n'est soumis à aucun contrôle gouvernemental. Il existe un marché traditionnel dans ce domaine, soumis à diverses réglementations : les structures doivent être agréées et répondre à certains critères en termes de qualifications du personnel, de ratio personnel/enfant, etc. (West et al., 2010).

7 Pour les parents à faibles revenus, il existe des aides gouvernementales en matière de garde d'enfants (via une prestation accordée par l'État sous le nom de Working Tax Credit ou «crédit d'impôt aux personnes en activité »). Sous le gouvernement de coalition, $70 \%$ des frais sont pris en charge (jusqu'à concurrence d'un certain plafond), si les parents ont recours à des structures de garde d'enfants certifiées et agréées. Si les parents travaillent et paient pour faire garder leurs enfants, l'employeur doit prendre en charge une partie de ces frais via un système de chèques-garderie, grâce auquel les frais de garderie sont prélevés sur le salaire brut avant déduction d'impôt et d'assurance nationale (HMRC 1 , 2013).

8 Lorsque les travaillistes étaient au pouvoir entre 1997 et 2010, la question de l'enseignement et des structures d'accueil destinés à la petite enfance constituait un enjeu politique fort. Précédemment, les conservateurs avaient mis en place un système de chèques-éducation pour financer la scolarisation à temps partiel des enfants de 4 ans. Lorsque les travaillistes furent élus en 1997, ils remplacèrent ce système de chèques-éducation par un mécanisme de financement axé sur la demande, par lequel les structures privées d'enseignement et d'accueil de la petite enfance (à but lucratif ou non lucratif) étaient financées sur la base du nombre d'enfants inscrits; les classes et les écoles maternelles (faisant partie du secteur financé par le public) étaient, elles, financées sur la base du nombre de places disponibles. 
9 L'offre éducative gratuite destinée à la petite enfance s'est élargie et à l'heure actuelle, tous les enfants âgés de 3 à 4 ans ont droit en Angleterre à 15 heures de prise en charge éducative gratuite, chaque semaine, pendant les 38 semaines que compte l'année scolaire (soit 570 heures par an, c'est-à-dire trois heures par jour, cinq jours par semaine). Cette prise en charge peut être assurée dans différentes structures, notamment dans les classes et les écoles maternelles rattachées aux écoles primaires et dans les garderies privées, à but lucratif ou non lucratif (voir West et al., 2010). Certains enfants âgés de 2 ans, dont les parents sont issus principalement de milieux socioéconomiques défavorisés (et généralement bénéficiaires de certaines allocations familiales), ont également accès à cette prise en charge.

L'offre éducative gratuite destinée à la petite enfance est financée par le gouvernement central au moyen d'une subvention spéciale, la Dedicated Schools Grant. Le montant réellement affecté à l'offre éducative destinée à la petite enfance, contrairement à celui affecté aux établissements scolaires, est déterminé par l'autorité éducative locale. Le financement de l'offre destinée à la tranche d'âge des 3-4 ans est attribué aux établissements en appliquant une formule déterminée par l'autorité locale, conformément aux textes réglementaires. Il s'agit de la «formule unique de financement de l'offre éducative destinée à la petite enfance ", qui doit inclure un taux de base unique valable pour toutes les structures ou des taux de base différenciés selon le type de structure, par rapport à des différences de coût jugées inévitables. La formule doit également inclure un supplément qui prenne en compte les difficultés socioéconomiques des familles et, pour toutes les structures concernées, elle doit être basée sur le décompte effectué au moins trois fois dans l'année des enfants bénéficiant de cette offre (Ministère de l'éducation, 2013).

\section{Ce que les familles paient}

11 Les familles paient des frais liés à la participation de leurs enfants à l'offre éducative préscolaire en sus de la part financée par le gouvernement. Ceci est important dans la mesure où l'offre éducative gratuite destinée à la petite enfance s'adresse aux enfants âgés de 3-4 ans (et aux enfants de 2 ans issus de milieux défavorisés) et est limitée dans le temps. De ce fait, cette offre est insuffisante pour assurer à elle seule la prise en charge des enfants, si les parents travaillent plus qu'à temps partiel.

\section{L'enseignement primaire et secondaire}

\section{Les établissements scolaires accueillant les 5-16 ans}

Environ $7 \%$ des élèves en Angleterre fréquentent des établissements privés payants (établissements dits "indépendants ", parfois appelés public schools). Les parents paient généralement des frais élevés pour que leurs enfants soient scolarisés dans ces établissements, dont beaucoup opèrent une forte sélection fondée sur les résultats scolaires. Ces établissements sont, dans leur quasi-totalité, à but non lucratif et jouissent d'un statut caritatif (charitable status). Selon le Charities Act (Loi sur les associations caritatives) de 2011, une charity se définit comme étant une institution créée à des fins caritatives et reconnue d'utilité publique. La promotion de l'éducation 
constituant un but caritatif, les établissements privés payants peuvent recevoir le statut d'association caritative et, à ce titre, bénéficier de divers avantages fiscaux.

La grande majorité des enfants en Angleterre (93\%) fréquentent des établissements publics. Il est important de noter que la plupart des établissements à profil religieux (principalement les établissements appelés « voluntary-aided ${ }^{2} »$ ) font partie du système éducatif financé par le public. Néanmoins, ils ont davantage d'autonomie que les établissements placés sous la tutelle administrative des autorités éducatives locales, par exemple sur le plan du recrutement du personnel et de l'enseignement de la religion (que les textes rendent obligatoire dans tous les établissements financés par le secteur public). Le recrutement des élèves s'effectue généralement sur des critères liés à la religion (Allen et West, 2011).

Des réformes axées sur le marché ont été introduites par la Loi sur l'éducation de 1980, qui a accordé une plus grande priorité aux choix des parents, ainsi que par la Loi sur la réforme de l'éducation de 1988, qui a eu pour effet de déterminer le financement des établissements scolaires principalement sur la base du nombre d'élèves inscrits et de contraindre les établissements à accueillir un nombre maximal d'élèves. Ces réformes ont également imposé la publication des résultats des élèves aux tests de contrôle et aux examens. L'un des objectifs de la politique était d'améliorer la qualité de l'enseignement dispensé, en favorisant les choix parentaux et la concurrence entre les établissements.

Depuis 2010, l'enseignement secondaire s'est transformé, avec le développement rapide des academies, structures scolaires gérées par des sociétés privées (à but non lucratif) et financées directement par une agence du gouvernement central (l'Education Funding Agency, l'agence de financement de l'éducation) sur la base d'un contrat ayant force obligatoire (dit « accord de financement »).

Ainsi coexistent aujourd'hui des établissements financés par les autorités éducatives locales et d'autres financés directement par le gouvernement central. Il est important de souligner que tous les établissements financés par le public sont gérés par leur chef d'établissement et leur conseil d'administration. Ce sont eux qui décident de la façon dont le budget doit être dépensé.

\section{Les établissements financés par les autorités éducatives locales}

17 Pour les établissements placés sous la tutelle administrative des autorités éducatives locales (cela ne concerne donc pas les academies), le gouvernement central attribue des fonds aux autorités locales qui les reversent ensuite aux établissements. Le système utilisé pour attribuer les ressources aux autorités locales a connu un certain nombre de changements ces dernières années. Avant 2006-2007, les autorités locales n'étaient pas contraintes de dépenser un montant donné pour l'éducation. Toutefois, depuis lors, le gouvernement attribue aux autorités locales une subvention fléchée pour financer l'éducation en milieu scolaire.

18 Les autorités locales fixent le budget alloué à l'éducation dans chacune de leur zone respective, pour chaque année scolaire, et reversent les fonds aux établissements dont elles ont la responsabilité. Elles assurent le financement de l'enseignement aux enfants et aux adolescents jusqu'à l'âge de 16 ans. Il est important de souligner que les autorités locales ne gèrent pas les établissements, mais en assurent le fonctionnement en attribuant les fonds et en fournissant un certain nombre de services réglementaires. $\mathrm{Ce}$ 
sont également elles qui emploient officiellement les enseignants (dans la plupart des types d'établissement dont elles ont la responsabilité).

19 Les autorités locales ont des devoirs et des pouvoirs spécifiques. En matière d'affectation des ressources aux établissements, elles ont l'obligation réglementaire de recourir à une formule fondée principalement sur le nombre d'élèves et leur âge (la formule doit prendre en compte un facteur lié à l'appartenance à un milieu défavorisé). Les formules varient selon les autorités locales. En ce qui concerne les dépenses en capital, les voluntary-aided schools, à profil principalement religieux, doivent contribuer à hauteur de $10 \%$ des coûts. Les établissements décident de la façon dont ils dépenseront l'argent qui leur est attribué et du recrutement des enseignants.

20 L'Education Funding Agency (l'agence gouvernementale précitée) est responsable du financement de l'offre éducative et de formation pour les 16-19 ans dans les établissements scolaires (ainsi que dans les collèges de formation complémentaire ${ }^{3}$ et les établissements privés). L'Education Funding Agency attribue les ressources aux autorités locales, elles-mêmes chargées de les reverser aux établissements (en dehors des academies) qui accueillent les 16-19 ans. La méthode de financement utilisée garantit un mode de calcul cohérent à l'échelle nationale pour le financement de tous les établissement dispensant une offre éducative et de formation aux 16-19 ans, en prenant en compte les facteurs suivants: nombre d'élèves, taux national de financement par élève, rétention scolaire, pondération du coût de la scolarité selon la filière (générale ou professionnelle), indemnité compensatoire selon la zone géographique, et appartenance à un milieu défavorisé.

21 L'Education Funding Agency conclut des accords de subvention avec chaque autorité locale pour financer l'offre éducative qui correspond à sa compétence (sachant que les collèges de formation complémentaire et les établissements privés sont financés directement par l'Education Funding Agency). L'accord de subvention détermine les fonds que l'Agence a accepté de verser en contrepartie de l'offre éducative et de formation prévue (Education Funding Agency, 2013).

\section{Les Academies}

22 Les academies se distinguent des autres établissements scolaires sur le plan de leur statut juridique. En janvier 2013, 50 \% des établissements secondaires et $6 \%$ des écoles primaires avaient le statut d'academy. Le nombre de ces academies ne cesse d'augmenter. Elles ont été créées à l'origine, dans les années 2000, pour remplacer les établissements secondaires jugés peu performants, afin d'améliorer la qualité de l'enseignement. Chaque academy recevait le soutien du secteur privé à but lucratif et non lucratif. Au départ, ce soutien était d'ordre financier. Suite aux élections législatives de 2010, le gouvernement de coalition entre les conservateurs et les libéraux-démocrates a étendu le système des academies. La Loi sur les academies de 2010 a permis, sous certaines conditions, aux établissements publics du primaire et du secondaire de bénéficier par conversion de ce statut spécial (on les a alors appelés les converter academies). Cette loi a également permis la mise en place de deux nouveaux types d'établissements, tous deux relevant des academies: d'une part, les établissements dits «libres» (free schools), financés par le secteur public, s'adressant à des élèves de tous profils, créés par des associations locales ou des organismes confessionnels, des parents, des enseignants, des associations caritatives, des entreprises, des universités et des établissements privés payants; d'autre part, des collèges universitaires 
d'enseignement technique et des studio schools ${ }^{4}$ destinées aux 14-19 ans (les premiers soutenus par des universités et des employeurs, les secondes par des entreprises locales et des employeurs).

Tous les types d'academies appartiennent à des institutions fiduciaires à but non lucratif et sont financés par contrat («l'accord de financement») avec le ministère de l'éducation. Les academies n'ont pas l'obligation d'appliquer le curriculum national (elles doivent néanmoins adopter un curriculum suffisamment « large et équilibré »), ni de se conformer à la réglementation relative aux salaires des enseignants. Elles sont par ailleurs responsables du recrutement de leurs élèves (West et Bailey, 2013).

Les academies reçoivent un financement de l'Education Funding Agency. Le montant de ce financement équivaut à celui d'un établissement public financé par les autorités locales du même district. Toutefois, les academies reçoivent également un financement destiné à des services auparavant assurés par les autorités locales: lors de sa conversion vers le statut d'academy, un établissement voit ainsi son budget augmenter. Cela a son importance, dans un contexte des mesures d'austérité mises en place par le gouvernement de coalition à partir de 2010. Si l'éducation en milieu scolaire a pu bénéficier d'une certaine forme de protection, il n'a pas été possible de garantir des niveaux de financement suffisants pour chaque établissement. On peut considérer que les ressources supplémentaires dont bénéficient les établissements se convertissant en academies ont pu servir d'incitation à changer de statut, les établissements pouvant alors décider eux-mêmes de la façon dont ils entendaient utiliser au mieux ces ressources (West et Bailey, 2013).

\section{Le financement de l'enseignement supérieur}

Les universités et les autres établissements d'enseignement supérieur sont des entités indépendantes qui puisent leurs revenus à des sources diverses: fonds publics du Conseil de financement de l'enseignement supérieur en Angleterre, frais de scolarité des étudiants, revenu des fondations universitaires, fonds provenant d'entreprises, d'autres organismes du secteur public, fonds dédiés à la recherche provenant d'organismes caritatifs et des conseils de recherche au Royaume-Uni. Le Conseil de financement de l'enseignement supérieur en Angleterre est une agence gouvernementale qui distribue des fonds publics pour l'enseignement supérieur. Leur montant est fixé annuellement par le gouvernement. Une grande partie du budget de ce Conseil va aux universités et aux collèges universitaires, ainsi qu'à un certain nombre d'autres établissements (les collèges de formation complémentaire, par exemple), qui proposent des formations d'enseignement supérieur. Le Conseil attribue des fonds dédiés à l'enseignement, à la recherche, ainsi qu'au renforcement des liens avec le monde de l'entreprise et avec les membres de la collectivité. Il attribue également des fonds exceptionnels (des crédits fléchés et des subventions spéciales d'investissement) afin de soutenir le développement de l'infrastructure nationale et des fonds en capital pour les dépenses uniques comme la construction de bâtiments et l'acquisition d'équipement (Conseil de financement de l'enseignement supérieur en Angleterre, 2014).

Une méthode de financement reposant sur une formule est utilisée pour soutenir la recherche et l'enseignement. Pour la recherche, la méthode de financement permet d'affecter les fonds de manière ciblée là où la qualité de la recherche est réputée être la 
plus élevée. Le secteur de l'enseignement supérieur a fait l'objet d'évaluations périodiques de la qualité de la recherche: plusieurs campagnes d'évaluation de la recherche (Research Assessment Exercises) et, très récemment, l'instauration du cadre d'excellence pour la recherche (Research Excellence Framework). Suite à la mise en place de ces dispositifs d'évaluation, le financement de la recherche est désormais attribué aux établissements d'enseignement supérieur sur la base de l'évaluation de la qualité de leur recherche.

Le financement de l'enseignement a fait l'objet d'un changement radical : à partir de 2012-2013, le gouvernement de coalition a décidé de verser davantage de fonds publics directement aux étudiants, sous la forme de prêts accordés aux étudiants pour financer les frais de scolarité (voir ci-dessous), et de réduire les subventions à l'enseignement versés aux établissements par le Conseil de financement de l'enseignement supérieur en Angleterre. Si les étudiants contractent des emprunts, les frais de scolarité sont alors versés aux établissements d'enseignement supérieur par une agence gouvernementale, la Student Loans Company (société de prêts aux étudiants). Les familles peuvent par ailleurs régler ces frais directement auprès de l'établissement concerné.

Les universités dépendent clairement des frais de scolarité payés par les étudiants. Il existe toutefois une limite au nombre d'étudiants que peut recruter chaque université, le " contrôle du nombre d'étudiants ", car le gouvernement entend garder la maîtrise des prêts couvrant les frais de scolarité et les frais d'entretien et de coût de la vie qui sont financés par l'État. Néanmoins, depuis 2012-2013, le gouvernement permet également aux établissements de recruter autant d'étudiants qu'ils le souhaitent (audelà de la limite imposée par le " contrôle du nombre d'étudiants ») parmi ceux qui ont obtenu les résultats les plus élevés aux examens nationaux sanctionnant la fin du second cycle de l'enseignement secondaire.

Il existe également un budget fixe consacré à l'enseignement, axé sur des secteurs prioritaires, pour lesquels on considère que les seuls frais de scolarité ne permettent pas de couvrir l'ensemble des coûts. À compter de l'année 2013-2014, ce budget concerne notamment le financement de certaines disciplines à coût élevé, ainsi que des disciplines à coût très élevé dans le domaine de la science, de la technologie, de l'ingénierie et des mathématiques (Conseil de financement de l'enseignement supérieur en Angleterre, 2014).

\section{Ce que les familles paient}

Depuis 1998-1999, les étudiants qui entament à temps plein un cursus d'enseignement supérieur en Angleterre, au Pays de Galles et en Irlande du Nord, sont tenus de contribuer financièrement aux frais de scolarité (qui s'élevaient au départ à $1000 £$, soit 1200 euros) à hauteur de leurs revenus ou des revenus de leur familles (West et Barham, 2010). Les frais de scolarité des étudiants en Licence ont augmenté en 2006-2007 pour atteindre $3000 £$ (3 600 euros) et à nouveau en 2012-2013, passant à $9000 £$ (10 800 euros). Ces frais ne sont pas payables «d'avance». Depuis 2012, l'aide financière accordée aux étudiants comprend un prêt couvrant les frais de scolarité, un prêt couvrant les frais d'entretien, et de coût de la vie, ou bien une aide concernant ces frais d'entretien, si les revenus sont inférieurs à une certaine somme, ainsi que diverses bourses d'études octroyées par les universités et les collèges universitaires. Une fois 
diplômés, les jeunes commenceront à rembourser leur prêt lorsqu'ils gagneront $21000 £$ (25000 euros). Ce nouveau régime contraint les universités à octroyer des bourses aux étudiants issus de milieux défavorisés. Un plan national d'octroi de bourses a par ailleurs été mis en place par le gouvernement, dans le souci d'attirer davantage d'étudiants issus de milieux défavorisés dans l'enseignement supérieur.

\section{Synthèse}

31 Les idées néolibérales se sont imposées en Angleterre dans les politiques gouvernementales en matière d'éducation. En ce qui concerne l'enseignement et les structures d'accueil destinés à la petite enfance, il existe depuis longtemps un marché pour l'offre de ces services. L'offre éducative gratuite destinée à la petite enfance, instaurée pour la première fois par le gouvernement travailliste et étendue depuis par la coalition actuelle entre conservateurs et libéraux-démocrates, est financée en appliquant une méthode fondée sur la demande : l'argent versé aux établissements suit les élèves inscrits.

Dans l'enseignement primaire et secondaire, les établissements sont également financés en appliquant une méthode fondée sur la demande. Le financement est attribué par le gouvernement central aux autorités locales, qui le reversent aux établissements dont elles ont la responsabilité. En ce qui concerne les academies, le financement est attribué par une agence gouvernementale, l'Education Funding Agency.

Le processus de conversion des établissements en academies a eu pour effet de modifier radicalement le système éducatif en milieu scolaire, passant d'un système national géré localement par des autorités locales démocratiquement élues, à un système centralisé dans lequel le ministère de l'éducation établit des contrats ayant force obligatoire avec un nombre croissant d'établissements privés (à but non lucratif). La rapidité et l'ampleur de ce processus de privatisation (à savoir le transfert de responsabilité du secteur public vers des acteurs extérieurs à celui-ci) méritent d'être remarquées.

Dans l'enseignement supérieur, des changements importants ont eu lieu, mais de nature différente. La pratique des subventions accordées aux établissements d'enseignement supérieur a été en grande partie remplacée. Aujourd'hui, les étudiants (ou leurs familles) paient des frais de scolarité. Ils n'ont pas à les payer d'avance car ils peuvent contracter des emprunts pour couvrir ces frais. Ils peuvent également bénéficier de prêts couvrant les frais d'entretien et de coût de la vie durant leurs études. L'agence chargée de verser les frais de scolarité aux universités et d'octroyer aux étudiants les prêts et les bourses couvrant les frais d'entretien et de coût de la vie, est la Student Loans Company, une entité privée. La mise en place de prêts couvrant les frais de scolarité, d'entretien et de coût de la vie peut s'apparenter à une forme de « privatisation » dont les familles supportent le coût (cf. Hacker, 2004).

Les changements intervenus en matière de financement du système éducatif anglais sont profonds. Au niveau de l'enseignement préscolaire, l'offre éducative à destination de la petite enfance est proposée aux enfants âgés de 3 à 4 ans, ainsi qu'à certains 
enfants de moins de 2 ans issus de milieux défavorisés. Un "quasi-marché » s'est superposé au marché traditionnel établi de longue date. Dans l'enseignement primaire et secondaire, il existe un quasi-marché dans lequel le financement suit les élèves. Le processus de conversion des établissements en academies a entraîné une modification $\mathrm{du}$ régime de financement: une agence centrale du gouvernement attribue les ressources aux academies à la place des autorités locales. Dans l'enseignement supérieur, la mise en place de frais de scolarité élevés a entraîné un processus de privatisation dont les familles supportent le coût, l'État ne subventionnant plus les universités pour une grande part de l'enseignement qu'elles dispensent.

\section{BIBLIOGRAPHIE}

ALLEN R. \& WEST A. (2011): Why do faith secondary schools have advantaged intakes? The relative importance of neighbourhood characteristics, social background and religious identification amongst parents, British Educational Research Journal, 37, 4, 691-712.

Department for Education (2013): Draft statutory guidance for local authorities on the exercise of their duties relating to early education and childcare. London: Department for Education.

Education Funding Agency (2013): Overview of the 16 to 19 Funding Formula Funding full participation and study programmes for young people. http://goo.gl/wePZ8W

HACKER J. (2004): Privatizing risk without privatizing the welfare state: The hidden politics of social policy retrenchment in the United States, American Political Science Review, 98, 2, 243-260.

HARVEY D. (2005): A Brief History of Neoliberalism, Oxford: Oxford University Press.

Higher Education Funding Council for England (2014): Funding higher education, Bristol: HEFCE. http://goo.gl/pJq4pA

HM Revenue and Customs (2013): Working Tax Credit - Help with the costs of childcare, London: HMRC.

WEST A. (2009): Redistribution and financing schools in England under Labour: Are resources going where needs are greatest? Educational Management, Administration and Leadership, 37, 2, 158-179.

WEST A. \& BAILEY E. (2013): The development of the academies programme: "Privatising" schoolbased education in England 1986-2013, British Journal of Educational Studies, 61, 2, 137-159.

WEST A. \& BARHAM E. (2010) : L'impact de la crise financière sur la politique d'éducation supérieure au Royaume-Uni, Economies et sociétés, 43, 627-644.

WEST A., ROBERTS J. \& NODEN P. (2010): Funding early years education and care: Can a mixed economy of providers deliver universal high quality provision? British Journal of Educational Studies, 58, 2, 155-179. 


\section{NOTES}

1. Her Majesty's Revenue and Customs (HMRC) : département non-ministériel du gouvernement du Royaume-Uni, chargé de la collecte des taxes et du paiement de certains services fournis par l'État. (NdT) (Source : Wikipédia).

2. Voluntary-aided schools: l'adjectif «voluntary» renvoie au secteur privé à but non lucratif. L'adjectif «- aided» renvoie au fait que ces établissements reçoivent des aides de l'État (environ $90 \%$ des coûts en capital et $100 \%$ des coûts de fonctionnement). Ils sont généralement fondés par des églises ou des organisations caritatives et sont responsables de l'inscription des élèves ainsi que $\mathrm{du}$ recrutement et de la gestion du personnel, sous l'autorité de leur conseil d'administration. Ces établissements ne sont pas payants pour les élèves, même si les parents sont encouragés à verser des contributions. La fondation ou l'institution fiduciaire (trust) est généralement propriétaire des lieux (NdT) (Sources : M. Breuillard et A. Cole, L'École entre l'État et les collectivités territoriales : En Angleterre et en France, L'Harmattan, 2003 ; http://en.wikipedia.org/ wiki/Voluntary_aided_school).

3. En Angleterre, les collèges de formation complémentaire (Further Education Colleges) proposent des enseignements à partir de l'âge de 16 ans, permettant à quiconque d'obtenir des compétences ou des qualifications, qu'elles soient générales ou professionnelles. Ils englobent les 6th-form colleges qui permettent aux élèves de 16 à 18 ans de parachever leurs études secondaires, ainsi que les instituts de formation technique et professionnelle et les instituts de formation pour adultes. Further Education se distingue de Higher Education (enseignement supérieur, université). $(\mathrm{NdT})$

4. Les studio schools proposent un enseignement général ou professionnel mais à orientation pratique, fondé sur des projets. L'enseignement se fait en alternance avec des stages en partenariat avec les entreprises locales. (NdT) (Source : Department for Education, http://goo.gl/ C9SxB4)

\section{RÉSUMÉS}

Cet article s'intéresse aux sources de financement et aux mécanismes utilisés pour financer l'éducation en Angleterre. À tous les niveaux du système éducatif, de nouveaux mécanismes de financement ont été mis en place au cours des dernières décennies. Ces mécanismes varient, mais en règle générale, on applique des formules pour répartir l'argent public à tous les niveaux, que ce soit dans l'enseignement préscolaire, primaire, secondaire ou supérieur. Les changements de politique ont eu pour conséquence une hausse de la dépense publique pour l'enseignement préscolaire. En revanche, les familles contribuent aujourd'hui pour une plus grande part à l'enseignement supérieur, dans la mesure où les emprunts contractés par les étudiants pour payer les frais de scolarité remplacent désormais les subventions publiques accordées aux universités.

This paper focuses on the sources of finance and mechanisms used to finance education in England. Across all phases of education, a range of new financing mechanisms have been introduced in recent decades. These vary, but formulae are generally used to distribute public money at all levels - pre-school, primary, secondary and higher education. Policy changes have resulted in more public money being channelled to pre-school education, but greater 
contributions to higher education are now being made by families as loans for tuition fees replace government grants to universities.

Este artículo se interesa por las fuentes de financiación y por los mecanismos utilizados para financiar la educación en Inglaterra. En todos los niveles del sistema educativo, unos mecanismos nuevos de financiación fueron instaurados durante estas últimas décadas. Estos pueden variar pero, de manera general, se aplican fórmulas para repartir el dinero público entre los distintos niveles - enseñanza preescolar, primaria, secundaria o superior. Los cambios de política tuvieron como consecuencia un alza del gasto público para la enseñanza preescolar. En cambio, las familias contribuyen hoy en gran parte a la enseñanza superior en la medida en que los préstamos contratados por los estudiantes para pagar los derechos de inscripción se sustituyen ahora a las subvenciones públicas otorgadas antes a las universidades.

\section{INDEX}

Index géographique : Angleterre, Royaume-Uni

Mots-clés : coût de l'éducation, frais de scolarité, éducation préscolaire, enseignement primaire, enseignement secondaire, enseignement supérieur, financement, fonds privés, fonds publics, politique éducative, marché de l'éducation

Palabras claves : enseñanza primaria, costes educativos, derechos de inscripción, educación de la primera infancia, enseñanza secundaria, enseñanza superior, financiación, fondos privados, fondos públicos, política educacional, mercado de la educación

Keywords : educational costs, educational fees, early childhood education, primary education, secondary education, higher education, financing, private funds, public funds, educational policy, education market

\section{AUTEURS}

\section{ANNE WEST}

Anne West est professeure de politique éducative au sein du département de politique sociale de la London School of Economics and Political Science, où elle dirige le groupe de recherche en éducation. Son travail de recherche porte sur la politique éducative (en Angleterre et dans un contexte comparatif), le financement des établissements scolaires, la politique éducative dans le domaine de la petite enfance et le soutien des parents au financement des études de leurs enfants. Elle s'intéresse particulièrement aux questions d'équité et d'égalité des chances. Elle a publié de nombreux articles. 